\title{
Filtrations of right ideals related to projectivity of left ideals
}

\author{
Vlastimil Dlab and Claus Michael Ringel
}

[This paper is in final form and no version of it will be submitted for publication else-where].

Let $k$ be a field and $A$ a finite-dimensional $k$-algebra. Since the endomorphism ring of the right $A$-module $A_{A}$ is $A$ itself, one must be able to describe all properties of $A$, for example properties of left ideals of $A$, in terms of the right $A$-module $A_{A}$. The aim of the present note is to show that the projectivity of certain left ideals can be characterized by the existence of suitable filtrations of right ideals.

As an application, we deal with quasi-hereditary rings. They have been defined by Scott [S] using heredity chains of ideals, thus using an inductive procedure of enlarging algebras. In this way one deals with a total ordering $e_{1}, \ldots, e_{n}$ of a complete set of primitive idempotents, with $e_{n}$ being added last. But there is a reverse procedure based on investigations of Mirollo and Vilonen [MV], and described in [DR2]: there we construct $A$ from $\varepsilon_{2} A \varepsilon_{2}$ where $\varepsilon_{2}=e_{2}+e_{3}+\cdots+e_{n}$. We characterize quasi-hereditary algebras such that the class of modules with Weyl filtrations is closed under submodules in terms of the two recursive procedures. And we show that algebras which satisfy this and the opposite condition have global dimension at most 2. It follows that the deep algebras introduced in [DR3], as well as the peaked ones defined in this paper have global dimension at most 2.

\section{The main results}

Unless otherwise stated, modules will be (finitely generated) right $A$-modules. Let $\mathcal{M}$ be a set of $A$-modules. Given a module $X_{A}$, an $\mathcal{M}$-filtration of $X_{A}$ is a chain of submodules $0=X_{0} \subset X_{1} \subset \cdots \subset X_{t}=X$ such that for all $1 \leq i \leq t$, the module $X_{i} / X_{i-1}$ is isomorphic to a module in $\mathcal{M}$.

Let $N$ be the (Jacobson) radical of $A$. Let $e_{1}, \ldots, e_{n}$ be a complete set of primitive (and orthogonal) idempotents. Let $E(i)=E\left(e_{i}\right)$ be the simple $A$-module not annihilated by $e_{i}$; thus $E_{i} \cong e_{i} A / e_{i} N$. Let $P(i)=P\left(e_{i}\right)$ be a projective cover of $E(i)$; thus $P(i) \cong e_{i} A$. Given a primitive idempotent $e$, we denote by $\hat{e}(i)$ the maximal quotient of $P(i)$ of Loewy length at most 2, whose radical is a direct sum of copies of $E(e)$. The set of modules $\hat{e}(i)$, with $1 \leq i \leq n$, is denoted by $\hat{e}$. The number of composition factors (in a composition series) of a module $X$ which are isomorphic to $E(i)$ will be denoted by $\ell_{i}(X)$. We recall that a module is said to be torsionless provided it is isomorphic to a submodule of a projective module.

Theorem 1. Let e be a primitive idempotent of $A$. The following statements are equivalent:

(i) The left ideal $N e$ is a projective left module.

(ii) $A_{A}$ has an $\hat{e}$-filtration and $\operatorname{Ext}_{A}^{1}(E(e), E(e))=0$.

(ii') Every right ideal has an $\hat{e}$-filtration. 
(ii") Every torsionless module has an $\hat{e}$-filtration.

Let $\varepsilon_{i}=e_{i}+\cdots+e_{n}$ for $1 \leq i \leq n$, and $\varepsilon_{n+1}=0$. We denote by $\Delta(i)$ the largest factor module of $P(i)$ with all composition factors of the form $E(j)$, with $j \leq i$; thus $\Delta(i)=e_{i} A / e_{i} A \varepsilon_{i+1} A$. The set of modules $\Delta(i)$, with $1 \leq i \leq n$, is denoted by $\Delta$, note that these modules $\Delta(i)$ depend on the chosen ordering $e_{1}, \ldots, e_{n}$. Let $I_{i}=A \varepsilon_{n-i+1} A$, thus $0=I_{0} \subset I_{1} \subset \cdots \subset I_{n}=A$ is a saturated chain of idempotent ideals of $A$. Note that $\left(I_{i}\right)_{i}$ is a heredity chain if and only if first, $A_{A}$ has a $\Delta$-filtration, and second, $\ell_{i}(\Delta(i))=1$, for all $1 \leq i \leq n$ : in this case, $A$ is said to be quasi-hereditary. (In case that $A$ is quasihereditary, the $\Delta$-filtrations of a module $X$ are also called "Weyl filtrations" [PS]. Also, $X$ has a $\Delta$-filtration if and only if its filtration $0=X I_{0} \subseteq X I_{1} \subseteq \cdots \subseteq X I_{n}=X$ is "good" in the sense of [DR2]; this follows from Lemma $1^{*}$ in section 2.)

Theorem 2. Assume that $\left(I_{i}\right)_{i}$ is a heredity chain, where $I_{i}=A \varepsilon_{n-1+1} A$, and let $C_{i}=$ $\varepsilon_{i} A \varepsilon_{i}$. Then the following conditions are equivalent:

(i) $\varepsilon_{i} N e_{i}$ is a projective left $C_{i}-$ module, for $1 \leq i \leq n$,

$\left(i^{\prime}\right) \quad \varepsilon_{i+1} N e_{i}$ is a projective left $C_{i+1}-$ module, for $1 \leq i \leq n-1$,

(ii) $\operatorname{rad} \Delta($ i $)$ has a $\Delta$-filtration, for $1 \leq i \leq n$,

(ii') every right ideal has a $\Delta$-filtration,

(ii") every torsionless module has a $\Delta$-filtration,

(ii"') submodules of modules with a $\Delta$-filtration have a $\Delta$-filtration.

The left modules $\Delta^{*}(i)$ and $\Delta^{*}=\left\{\Delta^{*}(i) \mid 1 \leq i \leq n\right\}$ are defined similarly as $\Delta(i)$ and $\Delta$, namely: $\Delta^{*}(i)$ is the largest factor module of $P^{*}(i)$ with all composition factors of the form $E^{*}(j)$ with $j \leq i$, thus $\Delta^{*}(i)=A e_{i} / A \varepsilon_{i+1} A e_{i}$. The fact that $\left(I_{i}\right)_{i}$ is a heredity chain may be expressed in a similar way in terms of $\Delta^{*}$. In the next theorem we deal with those algebras $A$ such that both $A$ and its opposite satisfy the equivalent conditions of Theorem 2.

Theorem 3. Let $\left(I_{i}\right)_{i}$ be a heredity chain. Assume that any right ideal of $A$ has a $\Delta-$ filtration and that any left ideal of $A$ has a $\Delta^{*}$-filtration. Then gl.dim. $A \leq 2$.

Corollary 1. Deep quasi-hereditary algebras have global dimension at most 2 .

We recall that the quasi-hereditary algebra $A$ is said to be deep [DR3] if, for every $1 \leq$ $i \leq n$, both the right $A$-module $\operatorname{rad} \Delta(i)$ and the left $A$-module $\operatorname{rad} \Delta^{*}(i)$ are projective.

The proofs of these results will be given in section 2, 3, and 4 of the paper. Section 5 contains a construction of a class of quasi-hereditary algebras of global dimension 2 which we call the peaked algebras. These are examples of algebras $A$ such that both $A$ and $A^{\text {opp }}$ satisfy the conditions of Theorem 2 . 


\section{Preliminaries on filtrations of modules.}

First, let $\mathcal{M}$ be an arbitrary set of modules. We consider modules which have an $\mathcal{M}-$ filtration. It is sometimes necessary to arrange the various quotients occurring in a filtration. In order to be able to do so, we will use the following well-known lemma.

Lemma 1. Assume that some $M \in \mathcal{M}$ satisfies $\operatorname{Ext}_{A}^{1}\left(M^{\prime}, M\right)=0$ for all $M^{\prime} \in \mathcal{M}$. Let $\mathcal{M}^{\prime}=\mathcal{M} \backslash\{M\}$. If a module $X$ has an $\mathcal{M}$-filtration, then it has a submodule $X^{\prime}$ with an $\mathcal{M}^{\prime}$-filtration such that $X / X^{\prime}$ is a direct sum of copies of $M$.

Proof. Let $X^{\prime \prime}$ be a submodule of $X$ with an $\mathcal{M}$-filtration such that $X / X^{\prime \prime}$ belongs to $\mathcal{M}$. By induction, there is a submodule $X^{\prime \prime \prime}$ of $X^{\prime \prime}$ with an $\mathcal{M}^{\prime}$-filtration such that $X^{\prime \prime} / X^{\prime \prime \prime}$ is a direct sum of copies of $M$. Since $\operatorname{Ext}_{A}^{1}\left(X / X^{\prime \prime}, X^{\prime \prime} / X^{\prime \prime \prime}\right)=0$, there is a submodule $Y$ of $X$ with $Y \cap X^{\prime \prime}=X^{\prime \prime \prime}$ and $Y+X^{\prime \prime}=X$. If $X / X^{\prime \prime}$ belongs to $\mathcal{M}^{\prime}$, let $X^{\prime}=Y$; otherwise, let $X^{\prime}=X^{\prime \prime \prime}$.

Lemma $1^{\star}$. Assume that some $M \in \mathcal{M}$ satisfies $\operatorname{Ext}_{A}^{1}\left(M, M^{\prime}\right)=0$ for all $M^{\prime} \in \mathcal{M}$. Let $\mathcal{M}^{\prime}=M \backslash\{M\}$. If a module $Y$ has an $\mathcal{M}$-filtration, then it has a submodule $Y^{\prime}$ which is a direct sum of copies of $M$ such that $Y / Y^{\prime}$ has an $\mathcal{M}^{\prime}$-filtration.

Clearly, this is the dual assertion. Both results have been used by Cline-Parshall-Scott [CPS] for dealing with modules over quasi-hereditary rings, or, more generally, with objects in highest weight categories.

We will be interested to know whether submodules of modules with an $\mathcal{M}$-filtration again have $\mathcal{M}$-filtrations. The following is a useful criterion in this direction.

Lemma 2. Assume that for any $M \in \mathcal{M}$, every maximal submodule of $M$ has an $\mathcal{M}$ filtration. Then submodules of modules with an $\mathcal{M}$-filtration have an $\mathcal{M}$-filtration.

Proof. Let $0=X_{0} \subset X_{1} \subset \cdots \subset X_{t}=X$ be an $\mathcal{M}$-filtration of the module $X$, let $Y$ be a submodule of $X$. We claim that $Y$ has an $\mathcal{M}$-filtration. By induction on the length of $X / Y$, we may assume that $Y$ is a maximal submodule of $X$. Choose $i$ minimal with $X_{i} \nsubseteq Y$. Then $X_{i} \cap Y$ is a maximal submodule of $X_{i}$ containing $X_{i-1}$. By assumption, $X_{i} \cap Y / X_{i-1}$ has an $\mathcal{M}$-filtration. Using it, we may refine the filtration $0=X_{0} \subset \ldots X_{i-1} \subseteq X_{i} \cap Y \subset \cdots \subset X_{t} \cap Y=Y$ in order to obtain an $\mathcal{M}$-filtration for $Y$.

We return to the complete set $e_{1}, \ldots, e_{n}$ of primitive idempotents of $A$, and we denote $e=e_{1}$. We assume that $\operatorname{Ext}_{A}^{1}(E(e), E(e))=0$. Let $\mathcal{M}(e)=\{\hat{e}(i) \mid 2 \leq i \leq n\}$, and let $\overline{\mathcal{M}}(e)$ be the set of non-zero quotient modules of modules in $\mathcal{M}(e)$.

Lemma 3. $A$ module $X$ has an $\overline{\mathcal{M}}(e)$-filtration if and only if $H_{o} m_{A}(X, E(e))=0$.

Proof. If $M$ is in $\overline{\mathcal{M}}(e)$, then $\operatorname{Hom}_{A}(M, E(1))=0$. Thus, if $X$ has an $\overline{\mathcal{M}}(e)$-filtration, $\operatorname{Hom}_{A}(X, E(1))=0$. Conversely, assume $\operatorname{Hom}_{A}(X, E(1))=0$. We may assume $X \neq 0$, thus let $X^{\prime}$ be a maximal submodule of $X$. Then $X / X^{\prime} \cong E(j)$ for some $2 \leq j \leq n$. Let $X^{\prime \prime}=\operatorname{rad} X^{\prime}$. There are (uniquely determined) submodules $Y, Y^{\prime}$ of $X^{\prime}$ containing 
$X^{\prime \prime}$ such that $X^{\prime} / X^{\prime \prime}=Y / X^{\prime \prime} \oplus Y^{\prime} / X^{\prime \prime}$ with $Y^{\prime} / X^{\prime \prime}$ a direct sum of copies of $E(1)$, and $Y / X^{\prime \prime}$ a direct sum of various $E(i)$, with $2 \leq i \leq n$. We claim that $X / Y$ belongs to $\mathcal{M}(e)$. For, the submodule $X^{\prime} / Y$ of $X / Y$ is a direct sum of copies of $E(1)$, the quotient is $X / X^{\prime} \cong E(j)$, and $\operatorname{Hom}_{A}(X / Y, E(1))=0$, thus $X^{\prime} / Y=\operatorname{rad}(X / Y)$. On the other hand, $\operatorname{Hom}_{A}(Y, E(1))=0$, since otherwise $\operatorname{Ext}_{A}^{1}(E(1), E(1)) \neq 0$. By induction, $Y$ has an $\overline{\mathcal{M}}(e)$-filtration and thus $X$ has an $\overline{\mathcal{M}}(e)$-filtration.

The length of the module $X$ will be denoted by $\ell(X)$; hence $\ell(X)=\sum_{i=1}^{n} \ell_{i}(X)$. Let $s_{i}=\ell(\hat{e}(i))$.

Lemma 4. Assume that $X$ has an $\bar{M}(e)$-filtration. Then

$$
\ell(X) \leq \sum_{i=2}^{n} \ell_{i}(X) s_{i}
$$

moreover the following assertions are equivalent:

$$
\ell(X)=\sum_{i=2}^{n} \ell_{i}(X) s_{i}
$$

(ii) the module $X$ has an $\mathcal{M}(e)-$ filtration,

(iii) any $\overline{\mathcal{M}}(e)$-filtration of $X$ is an $\mathcal{M}(e)$-filtration.

Proof. Let $0=X_{0} \subset X_{1} \subset \cdots \subset X_{t}=X$ be an $\overline{\mathcal{M}}(e)$-filtration, with $X_{j} / X_{j-1} \cong$ $\hat{e}(\sigma(j)) / U_{j}$, where $U_{j} \subseteq \operatorname{rad} \hat{e}(\sigma(j))$, and $2 \leq \sigma(j) \leq n$. Clearly, for $2 \leq i \leq n$, the number $\ell_{i}(X)$ is just the number of $j$ 's with $\sigma(j)=i$. Thus

$$
\begin{aligned}
\ell(X) & =\sum_{j=1}^{t} \ell\left(X_{j} / X_{j-1}\right)=\sum_{j=1}^{t} \ell(\hat{e}(\sigma(j)))-\sum_{j=1}^{t} \ell\left(U_{j}\right) \\
& =\sum_{i=2}^{n} \ell_{i}(X) s_{i}-\sum_{j=1}^{t} \ell\left(U_{j}\right) \leq \sum_{i=2}^{n} \ell_{i}(X) s_{i},
\end{aligned}
$$

and we have equality if and only if all $U_{j}=0$, that is if and only if the given filtration is an $\mathcal{M}(e)$-filtration.

Lemma 5. Assume that $X$ has an $\mathcal{M}(e)$-filtration, and let $e^{\prime}$ be an idempotent of $A$ with $e A e^{\prime} \subseteq N$. Then also $X / X e^{\prime} A$ has an $\mathcal{M}(e)$-filtration. 
Proof. Since $\operatorname{Hom}_{A}\left(X e^{\prime} A, E(e)\right)=0$, the module $X e^{\prime} A$ has an $\overline{\mathcal{M}}(e)$-filtration according to Lemma 3. Since $X$ has an $\overline{\mathcal{M}}(e)$-filtration, also $X / X e^{\prime} A$ has one, and therefore $X$ has an $\overline{\mathcal{M}}(e)$-filtration passing through $X e^{\prime} A$. But by Lemma 4 , any $\overline{\mathcal{M}}(e)$-filtration is an $\mathcal{M}(e)$-filtration.

Lemma 6. Assume $X$ has an $\hat{e}$-filtration. Then there is a submodule $X^{\prime}$ of $X$ with an $\mathcal{M}(e)$-filtration such that $X / X^{\prime}$ is a direct sum of copies of $E(e)$.

Proof. Since $\operatorname{Ext}^{1}(E(e), E(e))=0$, we have $\operatorname{Ext}^{1}(\hat{e}(i), E(1))=0$ for all $1 \leq i \leq n$. Now we apply Lemma 1.

\section{Proof of Theorem 1.}

As before, we deal with a complete set $e=e_{1}, e_{2}, \ldots, e_{n}$ of primitive idempotents.

If the left ideal $N e$ is a projective left module, its indecomposable summands have to be of the form $A e_{i}$, with $2 \leq i \leq n$. Since $A e$ cannot be embedded into $N e$, but $\operatorname{Ext}_{A}^{1}(E(e), E(e))=0$.

We are going to establish the equivalence of assertions(i) and (ii) in Theorem 1 , so we may assume from the beginning that $\operatorname{Ext}_{A}^{1}(E(e), E(e))=0$.

Recall that the species $\mathcal{S}=\left(D_{i, i} M_{j}\right)_{i, j}$ of $A$ is defined as follows: $D_{i}$ is the division ring $e_{i} A e_{i} / e_{i} N e_{i}$, and ${ }_{i} M_{j}$ is the $D_{i}-D_{j}$-bimodule $e_{i} N e_{j} / e_{i} N^{2} e_{j}$. Let $d_{i}=\operatorname{dim}_{k} D_{i}, d_{i j}=$

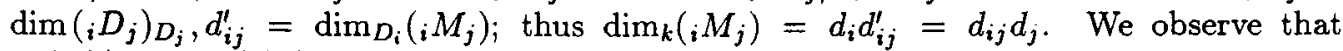
$\operatorname{rad} \hat{e}(i)=d_{i 1} E(1)\left(\right.$ thus $\left.s_{i}=d_{i 1}+1\right)$.

The simple left $A$-modules will be denoted by $E^{*}(i)=A e_{i} / N e_{i}$, their projective covers by $P^{*}(i)=A e_{i}$. The top of the left $A$-module $N e$ is isomorphic to $\bigoplus_{i=1}^{n} d_{i 1}^{\prime} E^{*}(i)$, and we consider the projective cover $p:{ }_{A} P \longrightarrow{ }_{A} N e$ of left $A$-modules: here, ${ }_{A} P \cong \bigoplus_{i=1}^{n} d_{i 1}^{\prime} P^{*}(i)$. Actually, the assumption $\operatorname{Ext}_{A}^{1}(E(e), E(e))=0$ can be reformulated as ${ }_{1} M_{1}=0$; thus $d_{11}=0=d_{11}^{\prime}$. Let ${ }_{A} Y$ be the kernel of $p$.

We decompose $A_{A}=e^{\prime} A \oplus e^{\prime \prime} A$, where $e^{\prime} A$ is a direct sum of copies of $e A$, and $e A e^{\prime \prime} \subseteq N$. Let $X_{A}=e^{\prime} N \oplus e^{\prime \prime} A$, thus $X e=N e$, and $X e_{i}=A e_{i}=P^{*}(i)$ for $2 \leq i \leq n$. In particular, for $2 \leq i \leq n$, we have

$$
\operatorname{dim}_{k} P^{*}(i)=\operatorname{dim}_{k} X e_{i}=\ell_{i}(X) d_{i}
$$

therefore

$$
\operatorname{dim}_{k} P=\sum_{i=2}^{n} d_{i 1}^{\prime} \operatorname{dim}_{k} P^{*}(i)=\sum_{i=2}^{n} \ell_{i}(X) d_{i} d_{i 1}^{\prime}=\sum_{i=2}^{n} \ell_{i}(X) d_{i 1} d_{1} .
$$

Since $\operatorname{Ext}_{A}^{1}(E(e), E(e))=0$, we have $\operatorname{Hom}_{A}\left(X_{A}, E(e)\right)=0$. Hence Lemma 3 asserts that $X_{A}$ has an $\overline{\mathcal{M}}(e)$-filtration, say $0=X_{0} \subset X_{1} \subset \cdots \subset X_{t}=X$ with $X_{j} / X_{j-1} \cong \hat{e}(\sigma(j)) / U_{j}$ 
for some submodule $U_{j}$ of $\hat{e}(\sigma(j))$ and $2 \leq \sigma(j) \leq n$. The number of $j$ 's with $\sigma(j)=i$ is $\ell_{i}(X)$. Since

$$
\ell_{1}\left(X_{j} / X_{j-1}\right)=\ell_{1}(\hat{e}(\sigma(j)))-\ell_{1}\left(U_{j}\right)=d_{\sigma(j), 1}-\ell\left(U_{j}\right)
$$

we have

$$
\begin{aligned}
\operatorname{dim}_{k} X e & =\sum_{j=1}^{t} \ell_{1}\left(X_{j} / X_{j-1}\right) d_{1}=\sum_{j=1}^{t}\left(d_{\sigma(j), 1}-\ell\left(U_{j}\right)\right) d_{1} \\
& =\sum_{i=1}^{n} \ell_{i}(X) d_{i 1} d_{1}-\sum_{j=1}^{t} \ell\left(U_{j}\right) d_{1}
\end{aligned}
$$

Comparing the dimensions of $P$ and $N e=X e$, we obtain the dimension for the kernel $Y$ of $p$

$$
\operatorname{dim}_{k} Y=\sum_{j=1}^{t} \ell\left(U_{j}\right) d_{1} .
$$

If we assume that ${ }_{A} N e$ is a projective left $A$-module, then $p$ is bijective, thus $Y=0$. Therefore all $U_{j}=0$, and our $\overline{\mathcal{M}}(e)$-filtration of $X_{A}$ is an $\mathcal{M}(e)$-filtration. Since $A_{A} / X_{A}$ is a direct sum of copies of $E(e)=\hat{e}(1)$, we conclude that $A_{A}$ has an $\hat{e}$-filtration.

Conversely, assume that $A_{A}$ has an $\hat{e}$-filtration. According to Lemma 6 , we obtain a submodule $\bar{X}_{A}$ of $A_{A}$ with an $\mathcal{M}(e)$-filtration such that $A_{A} / \bar{X}_{A}$ is a direct sum of copies of $E(e)$. Clearly, $\bar{X}_{A}=X_{A}$, so $X_{A}$ has an $\mathcal{M}(e)$-filtration. It follows that $U_{j}=0$ for all $j$, consequently $p$ is bijective, and therefore ${ }_{A} N e$ is a projective left $A$-module.

This shows the equivalence of assertions (i) and (ii). Every module $\hat{e}(i)$ in $\hat{e}$ has a unique maximal submodule, and this submodule is a direct sum of copies of $\hat{e}(1)=E(e)$. Hence, it has an $\hat{e}$-filtration. Lemma 1 asserts that submodules of modules with $\hat{e}$-filtrations have $\hat{e}$-filtrations. Under the assumption of (ii), any free module has an $\hat{e}$-filtration, thus any torsionless module has an $\hat{e}$-filtration. This shows $(\mathrm{ii}) \Rightarrow\left(\mathrm{ii}^{\prime \prime}\right)$, and trivially $\left(\mathrm{ii}^{\prime \prime}\right) \Rightarrow\left(\mathrm{ii}^{\prime}\right)$.

Finally, we show the implication (ii') $\Rightarrow$ (ii). Take a right ideal $Y_{A}$ of minimal length having $E(e)$ as a composition factor.

Clearly, $Y_{A}$ has a unique maximal submodule $Y^{\prime}$, and $Y / Y^{\prime} \cong E(e)$, whereas $Y^{\prime}$ has no composition factor of the form $E(e)$. Take an $\hat{e}$-filtration $0=Y_{0} \subset Y_{1} \subset \cdots \subset Y_{t}=Y$ of $Y$. Then $Y_{t-1} \subseteq Y^{\prime}$, and $Y^{\prime} / Y_{t-1}=\operatorname{rad}\left(Y_{t} / Y_{t-1}\right)$. Since $Y / Y^{\prime} \cong E(e)$, we see that $Y_{t} / Y_{t-1} \cong \hat{e}(1)$. Since $Y^{\prime} / Y_{t-1}$ has no composition factor $E(1)$, it follows that $Y^{\prime} / Y_{t-1}=0$. Thus $\hat{e}(1)=E(e)$, and therefore $\operatorname{Ext}_{A}^{1}(E(e), E(e))=0$. 


\section{Proof of theorem 2 .}

We assume that $\left(I_{i}\right)_{i}$ is a heredity chain, where $I_{i}=A \varepsilon_{n-i+1} A$, with $\varepsilon_{i}=e_{i}+\cdots+e_{n}$, for $1 \leq i \leq n$, and $\varepsilon_{n+1}=0$, and we denote $C_{i}=\varepsilon_{i} A \varepsilon_{i}$.

Lemma 7. The left ideal ${ }_{A} N e_{1}$ is a projective left $A$-module if and only if $\varepsilon_{2} N e_{1}$ is a projective left $C_{2}-$ module.

Proof. First, assume that ${ }_{A} N e_{1}$ is projective. Then ${ }_{A} N e_{1}$ is isomorphic to a module of the form $\bigoplus_{i=2}^{n} m_{i} A e_{i}$, for some $m_{i} \in \mathbf{N}_{0}$, since $A e_{1}$ cannot be embedded into $N e_{1}$. Thus $\varepsilon_{2} N e_{1} \cong \bigoplus_{i=2}^{n} m_{i}\left(\varepsilon_{2} A e_{i}\right)$, as a left $C_{2}$-module. But $\varepsilon_{2} A e_{i}$ is a projective left $C_{2}-$ module for $2 \leq i \leq n$, since $\varepsilon_{2}=e_{2}+\cdots+e_{n}$ with orthogonal idempotents $e_{2}, \ldots, e_{n}$.

Conversely, assume $\varepsilon_{2} N e_{1}$ is a projective left $C_{2}-$ module. Since $A \varepsilon_{2} A$ belongs to a heredity chain, we know that the multiplication map

$$
A \varepsilon_{2} \otimes_{C_{2}} \varepsilon_{2} A \longrightarrow A \varepsilon_{2} A
$$

is bijective (Prop. 7 of [DR2]). Multiplying from the right by $e_{1}$, we obtain an isomorphism $A \varepsilon_{2} \otimes \varepsilon_{2} A e_{1} \cong A \varepsilon_{2} A e_{1}$ of left $A$-modules. Since $A \varepsilon_{2}$ is a projective left $A$-module, and $\varepsilon_{2} A e_{1}=\varepsilon_{2} N e_{1}$ is a projective left $C_{2}$-module, it follows that $A \varepsilon_{2} A e_{1}$ is a projective left $A$-module. It remains to be shown that $A \varepsilon_{2} A e_{1}=N e_{1}$. First of all, $\varepsilon_{2} A e_{1} \subseteq N$, thus $A \varepsilon_{2} A e_{1}=A \varepsilon_{2} N e_{1}$. Second, $e_{1} N e_{1}=e_{1} N^{2} e_{1}$, thus the left $A$-module $N e_{1}$ is generated by $A \varepsilon_{2}$, consequently $A \varepsilon_{2} N e_{1}=N e_{1}$.

Note that the left $A$-module $N e_{1}$ is projective if and only if the left $C_{1}$-module $\varepsilon_{1} N e_{1}$ is projective. This an immediate consequence of the Morita equivalence of $A$ and $C_{1}$.

The equivalence of the assertions (i) and $\left(i^{\prime}\right)$ in Theorem 1 is an immediate consequence of Lemma 7: we apply it to the rings $C_{i}$ and their corresponding heredity chains ([DR1], statement 10$)$. The implication (ii) $\Rightarrow\left(\mathrm{ii}^{\prime \prime \prime}\right)$ is asserted in Lemma 2. Since $A_{A}$ has a $\Delta$-filtration, the same is true for any free $A$-module, thus (ii"') $\Rightarrow$ (ii"). The implications $\left(\mathrm{ii}^{\prime \prime}\right) \Rightarrow\left(\mathrm{ii}^{\prime}\right)$ is trivial. In order to prove the implication $\left(\mathrm{ii}^{\prime}\right) \Rightarrow$ (ii), we assume that the right ideals $e_{i} N$ have $\Delta$-filtrations. Then there are $\Delta$-filtrations of $e_{i} N$ passing through $e_{i} N \varepsilon_{i+1} A$, and therefore also $\operatorname{rad} \Delta(i)=e_{i} N / e_{i} N \varepsilon_{i+1} A$ has a $\Delta$-filtration.

It remains to verify the equivalence of the conditions (i) and (ii). We will use induction on $n$. The algebra $C_{2}$ has the heredity chain $0=\varepsilon_{2} I_{0} \varepsilon_{2} \subset \varepsilon_{2} I_{1} \varepsilon_{2} \subset \cdots \subset \varepsilon_{2} I_{n-1} \varepsilon_{2}=C_{2}$, and for $C_{2}$, we deal with the modules $\Delta_{2}(i)=e_{i} A \varepsilon_{2} / e_{i} A \varepsilon_{i+1} A \varepsilon_{2}=\Delta(i) \varepsilon_{2}$, where $2 \leq i \leq n$.

First, we assume that $\operatorname{rad} \Delta(i)$ has a $\Delta$-filtration, for $1 \leq i \leq n$. Then $\operatorname{rad} \Delta_{2}(i)$ has a $\Delta_{2}$-filtration, for $2 \leq i \leq n$, thus, by induction, $\varepsilon_{i} N e_{i}$ is a projective left $C_{i}$-module, for $2 \leq i \leq n$. We want to show that $N e_{1}$ is a projective left $A$-module. According to 
Theorem 1, it suffices to show that $A_{A}$ has an $\hat{e}$-filtration where $e=e_{1}$. Now $A_{A}$ has a $\Delta$-filtration, so we use the following lemma.

Lemma 8. Assume that rad $\Delta(i)$ has a $\Delta$-filtration, for all $1 \leq i \leq n$. Then any module with a $\Delta$-filtration has an $\hat{e}$-filtration.

Proof. Let $X$ be a module with a $\Delta$-filtration. We use induction on $\ell(X)$. We may assume $X=\Delta(i)$ for some $i$. If $\ell(\Delta(i))=1$, then $\operatorname{Ext}_{A}^{1}(E(i), E(j))=0$ for all $j \leq i$; in particular, $\operatorname{Ext}_{A}^{1}(E(i), E(1))=0$. Hence $\hat{e}(i)=E(i)=\Delta(i)$. Now assume $\ell(\Delta(i))>1$. Let $X=\operatorname{rad} \Delta(i)$. By induction, $X$ has an $\hat{e}$-filtration, thus there is a submodule $X^{\prime}$ with an $\mathcal{M}(e)$-filtration such that $X / X^{\prime}$ is a direct sum of copies of $E(1)$. It follows that $X^{\prime}=e_{i} N \varepsilon_{2} A$, thus $\Delta(i) / X^{\prime}=\hat{e}(i)$. Since $X^{\prime}$ has an $\hat{e}$-filtration, we see that $\Delta(i)$ has an $\hat{e}$-filtration.

Finally, we verify the implication (i) $\Rightarrow$ (ii). For $1 \leq i \leq n$, let $\varepsilon_{i} N e_{i}$ be a projective left $C_{i}$-module. By induction we know that $\operatorname{rad} \Delta_{2}(i)$ has a $\Delta_{2}$-filtration, for $2 \leq i \leq n$. Since $N e_{1}$ is a projective left $A$-module, Theorem 1 asserts that $A_{A}$ has an $\hat{e}$-filtration. We are going to show that $\operatorname{rad} \Delta(j)$, with $1 \leq j \leq n$, has a $\Delta$-filtration. Since $\Delta(1)=E(1)$, we may assume $2 \leq j \leq n$. Consider $Z_{j r}=(\operatorname{rad} \Delta(j)) \varepsilon_{r} A /(\operatorname{rad} \Delta(j)) \varepsilon_{r+1} A$, with $1 \leq r \leq n$. We claim that $Z_{j r}$ is a direct sum of copies of $\Delta(r)$. Again the case $r=1$ is trivial, so assume $2 \leq r \leq n$. First of all, top $Z_{j r}$ is clearly a direct sum of copies of $E(r)$, say top $Z_{j r}=$ $m_{j r} E(r)$. Since $\Delta(r)$ is the projective $A / A \varepsilon_{r+1} A$-cover of $E(r)$, and $Z_{j r}$ is annihilated by $A \varepsilon_{r+1} A$, it follows that there is a surjective map $Y \longrightarrow Z_{j r}$ with $Y=m_{j r} \Delta(r)$. In order to show that this is an isomorphism, we are going to prove that $\ell(Y)=\ell\left(Z_{j r}\right)$. First, we claim that both $Y$ and $Z_{j r}$ have $\mathcal{M}(e)$-filtrations. For, $e_{r} A$ has an $\hat{e}$-filtration, and $\operatorname{Hom}_{A}\left(e_{r} A, E(1)\right)=0$, since $r \geq 2$; thus $e_{r} A$ has an $\mathcal{M}(e)$-filtration by Lemma 6 . According to Lemma 5, $\Delta(r)=e_{r} A / e_{r} A \varepsilon_{e+1} A$ has an $\mathcal{M}(e)$-filtration, thus the same is true for $Y$. Since $\Delta(j)$ has an $\hat{e}$-filtration, also $\Delta(j) e_{r} A$ has one, according to Lemma 2. Using again $r \geq 2$, Lemma 5 and Lemma 6 , we see that $Z_{j r}$ has an $\mathcal{M}(e)$-filtration. Given any $A$-module $X$, and $i \geq 2$, the number $\ell_{i}(X)$ coincides with the number $\ell^{(2)}\left(X \varepsilon_{2}\right)$ of composition factors of the $C_{2}$-module $X \varepsilon_{2}$ which are of the form $E(i) \varepsilon_{2}=e_{i} A \varepsilon_{2} / e_{i} N \varepsilon_{2}$. We use Lemma 4 in order to express $\ell(Y)$ and $\ell\left(Z_{j r}\right)$ as follows:

$$
\begin{gathered}
\ell(Y)=\sum_{i=2}^{n} \ell_{i}(Y) s_{i}=\sum_{i=2}^{n} \ell_{i}^{(2)}\left(Y \varepsilon_{2}\right) s_{i} \\
\ell\left(Z_{j r}\right)=\sum_{i=2}^{n} \ell_{i}\left(Z_{j r}\right) s_{i}=\sum_{i=2}^{n} \ell_{i}^{(2)}\left(Z_{j r} \varepsilon_{2}\right) s_{i}
\end{gathered}
$$

On the other hand,

$$
\begin{aligned}
Z_{j r} \varepsilon_{2} & =(\operatorname{rad} \Delta(j)) \varepsilon_{r} A \varepsilon_{2} /(\operatorname{rad} \Delta(j)) \varepsilon_{r+1} A \varepsilon_{2}= \\
& =\left(\operatorname{rad} \Delta_{2}(j)\right) \varepsilon_{r} C_{2} /\left(\operatorname{rad} \Delta_{2}(j)\right) \varepsilon_{r+1} C_{2}
\end{aligned}
$$

is a direct sum of copies of $\Delta_{2}(r)$, since $\Delta_{2}(j)$ has a $\Delta_{2}$-filtration. It follows that $Z_{j r} \varepsilon_{2} \cong$ $m_{j r} \Delta_{2}(r)=Y \varepsilon_{2}$. As a consequence, $\ell(Y)=\ell\left(Z_{j r}\right)$. This completes the proof of the implication (i) $\Rightarrow$ (ii). 


\section{Algebras of global dimension 2 .}

We are going to present the proof of Theorem 3 as well as some related examples. As before let $e_{1}, \ldots, e_{n}$ be a complete set of primitive and orthogonal idempotents, and let $\varepsilon_{i}=e_{1}+\cdots+e_{n}$ for $1 \leq i \leq n$. Again, we assume that $\left(I_{i}\right)_{i}$ is a heredity chain, where $I_{i}=A \varepsilon_{n-i+1} A$.

Lemma 9. Let $\varepsilon=\varepsilon_{2}$. Let $C=\varepsilon A \varepsilon$. Assume that $\varepsilon N e_{1}$ is a projective left $C$-module and that $e_{1} N$ is a projective right $A$-module. Then proj.dim. $E(1)_{A} \leq 1$, and proj.dim. $E(i)_{A} \leq$ $\max \{2, \operatorname{proj.dim} .(E(i) \varepsilon) C\}$ for $2 \leq i \leq n$.

Proof. Since $E(1)=e_{1} A / e_{1} N$, it follows that proj.dim. $E(1)_{A} \leq 1$. Consider now $E(i)$, where $2 \leq i \leq n$. We can assume that proj.dim. $(E(i) \varepsilon)_{C}$ is finite; let

$$
0 \longrightarrow P^{(m)} \longrightarrow \ldots \longrightarrow P^{(1)} \longrightarrow P^{(0)} \longrightarrow E(i) \varepsilon \longrightarrow 0
$$

be a projective resolution of the $C$-module $(E(i) \varepsilon)_{C}$. We tensor this sequence with $C(\varepsilon A)$. Note that $C(\varepsilon A)$ is a direct sum of copies of $C\left(\varepsilon A e_{j}\right)$, with $1 \leq j \leq n$. For $2 \leq j \leq n$, the left $C$-module $C\left(\varepsilon A e_{j}\right)$ is projective, since $e_{j}$ is an idempotent of $C$, and $C\left(\varepsilon A e_{1}\right)={ }_{C}\left(\varepsilon N e_{1}\right)$ is projective by assumption. Thus

$$
0 \longrightarrow P^{(m)} \otimes_{C} \varepsilon A \longrightarrow \ldots \longrightarrow P^{(0)} \otimes_{C} \varepsilon A \longrightarrow E(i) \varepsilon \otimes_{C} \varepsilon A \longrightarrow 0
$$

is exact. Since the $A$-modules $P^{(s)} \otimes C(\varepsilon A)$ are projective, it follows that proj.dim. $E(i) \varepsilon \otimes$ ${ }_{C}(\varepsilon A)_{A} \leq m$. The exact sequence $0 \longrightarrow e_{i} N \longrightarrow e_{i} A \longrightarrow E(i) \longrightarrow 0$ yields first by multiplying with $\varepsilon$ and then tensoring with $C(\varepsilon A)$, the exact sequence

$$
0 \longrightarrow e_{i} N \varepsilon \otimes_{C} \varepsilon A \longrightarrow e_{i} A \varepsilon \otimes_{C} \varepsilon A \longrightarrow E(i) \varepsilon \otimes_{C} \varepsilon A \longrightarrow 0 .
$$

Since $A \varepsilon A$ belongs to a heredity chain, we can identify $A \varepsilon \otimes_{C} \varepsilon A$ with $A \varepsilon A$ and therefore $e_{i} A \varepsilon \otimes_{C} \varepsilon A$ with $e_{i} A \varepsilon A=e_{i} A$. We see that $E(i) \varepsilon \otimes_{C} \varepsilon A \cong e_{i} A / e_{i} N \varepsilon A=\hat{e}(i)$. Thus proj.dim. $\hat{e}(i)_{A} \leq m$. There is the exact sequence

$$
0 \longrightarrow d_{i 1} E(1) \longrightarrow \hat{e}(i) \longrightarrow E(i) \longrightarrow 0
$$

Since proj.dim. $E(1) \leq 1$, it follows that

$$
\text { proj.dim. } E(i) \leq \max \left\{2, \operatorname{proj.dim.ê(}(i)_{A}\right\}=\max \{2, m\}
$$


Proof of Theorem 3. We use induction on $n$. Condition (i) of Theorem 2 applied to $A$ and to its opposite shows that $C=C_{2}$ satisfies the corresponding assumptions (every right ideal of $C_{2}$ has a $\Delta_{2}$-filtration, every left ideal of $C_{2}$ has a $\Delta_{2}^{*}$-filtration). Thus gl.dim. $C \leq 2$. Also, $\varepsilon_{2} N e_{1}$ is a projective left $C_{2}$-module by condition (i') of Theorem 2 . And $e_{1} N \varepsilon_{1}$ is a projective right $C_{1}$-module by condition (i) of Theorem 2, applied to the opposite of $A$, thus $e_{1} N$ is a projective $A$-module. We apply Lemma 9 and conclude that gl.dim $A \leq 2$.

Let us remark that not all algebras of global dimension 2 satisfy the conditions of Theorem 2: A simple example is provided by the path algebra of the graph

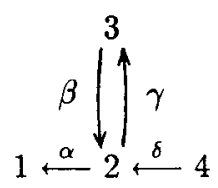

modulo the ideal $\langle\beta \alpha, \beta \gamma, \delta \gamma\rangle$ :

$$
A_{A}=1 \oplus 1 \quad \begin{aligned}
& 2 \\
& 3 \\
& 2
\end{aligned} \overbrace{2}^{3} \oplus \begin{aligned}
& 4 \\
& 2 \\
& 3 \\
& 2
\end{aligned}
$$

Here,

$$
\Delta(1)=1, \quad \Delta(2)=\begin{aligned}
& 2 \\
& 1
\end{aligned}, \quad \Delta(3)=\begin{aligned}
& 3 \\
& 2
\end{aligned}, \quad \Delta(4)=\begin{aligned}
& 4 \\
& 2 \\
& 3 \\
& 2
\end{aligned},
$$

thus $\operatorname{rad} \Delta(4)$ has no $\Delta$-filtration. On the other hand, the path algebra of

$$
1 \stackrel{\alpha_{1}}{\longrightarrow} 2 \stackrel{\alpha_{2}}{\longrightarrow} 3 \stackrel{\alpha_{3}}{\longrightarrow} \ldots \stackrel{\alpha_{n-1}}{\longrightarrow} n
$$

modulo $\left\langle\alpha_{i-1} \alpha_{i} \mid 2 \leq i \leq n-1\right\rangle$ satisfies the conditions of Theorem 2, but has global dimension $n-1$. Of course, for $n \geq 4$ this implies that its opposite algebra does not satisfy these conditions. Observe that, for $n=3$ this is an example of an algebra of global dimension 2 whose dimension (namely 5 ) is less than the dimension of the corresponding peaked algebra (of dimension 6) as defined in the next section. 


\section{Peaked algebras}

In this last section, we intend to give a construction of a new class of quasi-hereditary algebras of global dimension 2 which may be of further interest. Let $\mathcal{S}=\left(D_{i, i} M_{j}\right)_{1 \leq i, j \leq n}$ be a labelled species without loops [DR3]: thus ${ }_{i} M_{i}=0$ for all $i$, and the index set $\{1,2, \ldots, n\}$ is considered with its natural ordering. As in [DR3], let

$$
\begin{gathered}
T=T(n)=\left\{\left(t_{o}, t_{1}, \ldots, t_{m}\right) \mid 0 \leq t_{i} \leq n \text { are integers, } m \geq 1,\right. \text { and } \\
\left.t_{i-1} \neq t_{i} \text { for all } 1 \leq i \leq m\right\}
\end{gathered}
$$

for every $t=\left(t_{0}, t_{1}, \ldots, t_{m}\right) \in T$, let

$$
M(t)={ }_{t_{0}} M_{t_{1}} \otimes D_{t_{1}} t_{1} M_{t_{2}} \otimes D_{t_{2}} \cdots \otimes_{D_{t_{m-1}}} t_{m-1} M_{t_{m}}
$$

and for $T^{\prime} \subseteq T$, let

$$
M\left(T^{\prime}\right)=\bigoplus_{t \in T^{\prime}} M(t)
$$

We define the ideal $M\left(W^{0}\right)$ of the tensor algebra $\mathcal{T}(\mathcal{S})$ by specifying the subset $W^{0}$ of $T$ as follows:

$$
W^{0}=W^{0}(n)=\left\{\left(t_{0}, t_{1}, \ldots, t_{m}\right) \in T \mid \text { there is } 0<i<m \text { such that } t_{i-1}>t_{i}<t_{i+1}\right\}
$$

Let $W$ be the complement $T \backslash W^{0}$, thus

$$
\begin{aligned}
& W=\left\{\left(t_{0}, t_{1}, \ldots, t_{m}\right) \in T \mid \text { there is } 0 \leq i \leq m\right. \text { such that } \\
& \left.\qquad t_{0}<t_{1}<\cdots<t_{i}>\cdots>t_{m-1}>t_{m}\right\} .
\end{aligned}
$$

Hence

$$
[M(T)]^{2 n-1} \subseteq M\left(W^{0}\right) \subseteq M(T)
$$

and thus $M\left(W^{0}\right)$ is an admissible ideal. Let

$$
\mathcal{P}(\mathcal{S})=\mathcal{T}(\mathcal{S}) / M\left(W^{0}\right)
$$

Observe that the Loewy length of $\mathcal{P}(\mathcal{S})$ is at most $2 n-1$, and that, as an abelian group, $\mathcal{P}(\mathcal{S})$ can be identified with

$$
\prod_{i=1}^{n} D_{i} \oplus M(W)
$$

We call $\mathcal{P}(\mathcal{S})$ the peaked algebra with labelled species $\mathcal{S}$. 
Proposition. Let $\mathcal{P}(\mathcal{S})$ be the peaked algebra with labelled species $\mathcal{S}$. Then $\mathcal{P}(\mathcal{S})$ is quasihereditary, every right ideal of $\mathcal{P}(\mathcal{S})$ has a $\Delta$-filtration, every left ideal of $\mathcal{P}(\mathcal{S})$ has a $\Delta^{*}$-filtration. In particular, gl.dim. $\mathcal{P}(\mathcal{S}) \leq 2$.

Proof. For any $1 \leq i \leq n$, we claim that $\operatorname{rad} \Delta(i)$ is a direct sum of various $\Delta(j)$. Since $\Delta(1)$ is simple, we can assume $2 \leq i \leq n$. Let

$$
T_{i}=\left\{\left(i, t_{1}, \ldots, t_{m}\right) \in T \mid i>t_{1}>\cdots>t_{m}\right\}
$$

Then $\Delta(i)$ may be identified with $D_{i} \oplus M\left(T_{i}\right)$, thus

$$
\operatorname{rad} \Delta(i)=M\left(T_{i}\right)=\bigoplus_{\left(i, j, t_{2}, \ldots, t_{m}\right) \in T_{i}} d_{i j} \Delta(j)
$$

where, as before, $d_{i j}=\operatorname{dim}\left({ }_{i} M_{j}\right)_{D_{j}}$.

In comparison with the deep algebras over a given labelled species (whose global dimension is also at most 2), the dimensions of the peaked algebras are considerably smaller. For instance, for $\mathcal{S}_{n}=\left(D_{i},{ }_{i} M_{j}\right)_{1 \leq i, j \leq n}$, where $D_{i}=k$ for all $i$ and ${ }_{i} M_{j}={ }_{k} k_{k}$ for all $i \neq j$ and ${ }_{i} M_{i}=0$ for all $i$, the dimensions $p(n)$ of $\mathcal{P}\left(\mathcal{S}_{n}\right)$ clearly satisfy

$$
p(n+1)=p(n)+4^{n}
$$

and thus, for all $n$,

$$
p(n)=\frac{1}{3}\left(4^{n}-1\right)
$$

On the other hand, let $d(n)$ be the dimension of a deep algebra over $\mathcal{S}_{n}$. We have $d(5)=$ 3263441 while $p(5)=341$, and $d(10) \approx 2.7 \times 10^{208}(!)$ while $p(10)=349525$. Even $p(20)$ is "only" 366503875925.

\section{References}

[CPS] Cline, E., Parshall, B., and Scott, L.: Finite dimensional algebras and highest weight categories. J.Reine.Ang.Math. 391(1988), 85-99

[DR1] Dlab, V., and Ringel, C.M.: Quasi-hereditary algebras. Illinois J.Math. (to appear)

[DR2] Dlab, V., and Ringel, C.M.: A construction for quasi-hereditary algebras. Compositio Math. (to appear) 
[DR3] Dlab, V., and Ringel, C.M.: The dimension of a quasi-hereditary algebra. Proceedings Banach Center Warszawa (to appear)

[MV] Mirollo, R., and Vilonen, K.: Bernstein-Gelfand-Gelfand reciprocity on perverse sheaves. Ann.Scient.Ec.Norm.Sup.4 série 20(1987), 311-324

[PS] Parshall, B.J., and Scott, L.L.: Derived categories, quasi-hereditary algebras and algebraic groups. Proc. Ottawa-Moosonee Workshops in Algebra. Carleton Univ. Notes No.3(1988)

[S] Scott, L.L.: Simulating algebraic geometry with algebra I.: Derived categories and Morita theory. Proc. Symp.Pure Math., Amer.Math.Soc., Providence 47 (1987), part 1, 271-282.

V. Dlab

Department of Mathematics

Carleton University

Ottawa K1S 5B6

Canada

C.M. Ringel

Fakultät für Mathematik

Universität

D-4800 Bielefeld 1

West Germany 